\title{
Concentración y periodicidad en el control del crecimiento y desarrollo del niño menor de un año
}

\section{Concentration and periodicity in the control of the growth and development of the child under one year}

Eler Borneo Cantalicio', 2, a

\author{
Filiación y grado académico \\ 1 Universidad de Huánuco, Perú (Docente). \\ 2 Red de Salud de Huánuco. Perú \\ a Magíster Salud Pública (Enfermero). \\ (10) ORCID iD \\ https://orcid.org/0000-0002-6273-9818 \\ Fuente de financiamiento \\ Este estudio recibió financiamiento de la \\ Universidad de Huánuco. \\ Conflictos de interés \\ El autor forma parte del equipo editorial de \\ la Revista Peruana de Ciencias de la Salud, \\ reservándose de participar en el proceso de \\ revisión. \\ Recibido: 02/11/2018 \\ Arbitrado por pares \\ Aceptado: 04/02/2019 \\ Citar Como \\ Borneo E. Concentración y periodicidad en \\ el control del crecimiento y desarrollo del \\ niño menor de un año. Rev Peru Cienc Salud. \\ 2019; (1): 14-20. doi: https://doi.org/10.37711/ \\ rpcs.2019.1.1.2
}

Correspondencia

Eler Borneo Cantalicio

Dirección: Jr. San Luís Gonzaga №104-Amarilis Cel.: 956419046

Email: ebornec50@hotmail.com

\section{RESUMEN}

Objetivo. Analizar la relación entre la concentración y la periodicidad del control y el perfil de crecimiento y desarrollo del niño. Métodos. Se ha realizado un estudio de diseño correlacional con 309 niños menores de un año del Centro de Salud Aparicio Pomares, Huánuco, en el 2018. Se empleó una ficha de recolección de datos. En el análisis inferencial se utilizó la prueba de chi-cuadrada de Pearson. Resultados. Entre los recién nacidos, 74,2 \% (207) presentaron concentración de controles y 43,0 \% (120) mantuvieron la periodicidad de controles. Asimismo, 94,3\% (263) fueron diagnosticados con crecimiento adecuado y $100,0 \%$ (279) tuvieron desarrollo psicomotor normal. En niños de 01 a 11 meses de edad, 62,8 \% (194) mostraron concentración de controles y 13,9\% (43) tuvieron periodicidad de controles. Del mismo modo, 57,0\% (176) fueron diagnosticados con crecimiento adecuado y $97,7 \%$ (302) tuvieron un desarrollo psicomotor normal. Por otro lado, la concentración de controles se relaciona con el crecimiento de los recién nacidos, con un $\mathrm{p} \leq 0,004$; mientras que en niños menores de un año, la concentración de controles se relaciona con el crecimiento $(p \leq 0,003)$ $y$ el desarrollo psicomotor $(p \leq 0,014)$. Conclusión. La concentración de controles se relaciona significativamente con el crecimiento y desarrollo de niños menores de un año del Centro de Salud Aparicio Pomares, Huánuco.

Palabras clave: infante, recién nacido, Niño, Infantil, Recopilación de datos, Diseño de la investigación, Fenómenos biológicos, Metas, Crecimiento y desarrollo (fuente: DeCS BIREME).

\section{ABSTRACT}

Objective. Analyze the relationship between the concentration and the periodicity of the control and the growth and development profile of the child under one year. Methods. A correlational design study was carried out with 309 children under one year from the Aparicio Pomares - Huánuco health center, in 2018. A data collection sheet was used. In the inferential analysis, the Pearson Chi-Square7 Test was used. Results. Among the newborns, 74.2 \% (207) presented a concentration of controls and $43.0 \%$ (120) maintained the periodicity of controls. Likewise, $94.3 \%$ (263) were diagnosed with adequate growth and $100.0 \%$ (279) had normal psychomotor development. In children aged 1 to 11 months, $62.8 \%$ (194) showed a concentration of controls and $13.9 \%$ (43) had a periodicity of controls. Similarly, $57.0 \%$ (176) were diagnosed with adequate growth and $97.7 \%$ (302) had normal psychomotor development. On the other hand, the concentration of controls is related to the growth of newborns, with a $\mathrm{p} \leq 0.004$; while in children under one year of age, the concentration of controls is related to growth $(p \leq 0.003)$ and psychomotor development $(p \leq 0.014)$. Conclusions. The concentration of controls is significantly related to the growth and development of children under one year of the Aparicio Pomares - Huánuco health center.

Keywords: infant, Newborn, Child Infant, Data Collection, Research Design, Biological Phenomena, Goals, Growth and Development (source: MeSH-NLM). 


\section{INTRODUCCIÓN}

La desnutrición crónica (talla baja para la edad) corresponde a un indicador clave para la medición del bienestar en el niño, así como del progreso de una nación. Es una importante variable económica traducida en condiciones de salud, acumulación del capital humano y el nivel de pobreza (1). Asimismo, existe mayor probabilidad de morir durante los primeros cinco años de vida, es más proclive a la enfermedad y a desencadenar un peor desempeño escolar. A la vez, posee mínimas oportunidades en ingresos económicos en la etapa adulta y es más vulnerable a la obesidad y a daños no transmisibles ${ }^{(2)}$.

Cerca de 156 millones de niños menores de cinco años a nivel mundial se encontraban con retraso en el crecimiento en 2015; lo que representa el 23,2 \% de los menores en este grupo de edad ${ }^{(3)}$. En México la desnutrición es de 20,9\% en niños menores de 5 años, siendo prevalente en la zona rural. Los niños menores de un año presentan los porcentajes más altos de desnutrición crónica ${ }^{(4,5)}$.

Entre 1990 y 2010, en Colombia la prevalencia de la desnutrición crónica tiene una tendencia a disminuir en $-12,9 \%$ en el $2010(13,2 \%)$ en relación al año 1990 $(26,1 \%)$, sin embargo, no se ha logrado cumplir con los Objetivo de Desarrollo del Milenio que proponen reducirla al $8,0 \%{ }^{(6)}$.

Para el 2012 en el Ecuador, la prevalencia de desnutrición crónica en los primeros 5 meses de vida fue de $9,5 \%$ y de 6 a 11 meses de $19,9 \%{ }^{(7)}$.

En el Perú, según la Organización Mundial de la Salud, la prevalencia de desnutrición crónica en niños menores de cinco años ha descendido de $28,0 \%$, en el 2007, a 12,9\%, en el 2017. Sin embargo, en la Región Huánuco, para el año 2017, la prevalencia fue del $19,6 \%{ }^{\left({ }^{8}\right)}$.Y, según el Sistema de Información del Estado Nutricional (SIEN), para el año 2017, las prevalencias de desnutrición crónica en niños en menores de cinco años fueron del $18,4 \%, 12,1 \%$ y $10,6 \%$ para la provincia de Huánuco, distrito de Huánuco y Centro de Salud Aparicio Pomares, respectivamente ${ }^{(9)}$.

Se trata de un fenómeno de origen multifactorial, influido por los determinantes sociales y económicas, teniendo como causa directa la ingesta inadecuada de nutrientes y la prevalencia de las enfermedades de tipo infeccioso; especialmente las de tipo respiratorio y gastrointestinales ${ }^{(10)}$.

Asimismo, existen condiciones sociales íntimamente asociadas con su desarrollo, como son el bajo grado educativo de la progenitora; la nutrición deficiente en calidad y cantidad; las condiciones inadecuadas de salud y saneamiento, y el inferior nivel socioeconómico de la madre en la toma de decisiones al interior del hogar ${ }^{(11)}$.

Los daños ocasionados resultan permanentes e irrecuperables posterior al segundo año de vida; determinando el desarrollo del niño y el surgimiento de enfermedades degenerativas en la etapa adulta ${ }^{(12)}$.

Esta problemática acarrea como consecuencias a largo plazo como las asociadas al desarrollo conductual y cognitivo, la salud reproductiva y el rendimiento académico, debilitando así la futura productividad. Asímismo, otras consecuencias pueden ser la obesidad y las enfermedades crónicas, la misma que se perpetúa la pobreza tanto directamente, a través de una pérdida de productividad debido a la mala condición física, e indirectamente, a través de una función pobre cognitiva y falta de aprendizaje ${ }^{(13)}$.

Sin embargo, en los últimos años, la Organización de las Naciones Unidas (ONU) impulsa el cumplimiento de los Objetivos de Desarrollo Sostenible (ODS) a ser cumplidos en el año 2030 siendo el objetivo dos y tres orientados a mejorar la calidad de vida de las personas, en especial la de la niñez el mismo que consiste: dar fin al hambre y garantizar el acceso de todas las personas, a una alimentación nutritiva, sana y suficiente durante todo el año y poner fin a las muertes evitables de recién nacidos y de niños menores de 5 años ${ }^{(14)}$.

El Control del Crecimiento y Desarrollo infantil, como una actividad crítica en la supervivencia y desarrollo de la infancia, fue propuesto por primera vez en la década del 70 en Nigeria, por el médico británico David Morley, quien encontró que el $80 \%$ de la mortalidad infantil podía ser evitada y preservado, mediante intervenciones preventivas y promocionales desarrolladas en establecimientos de salud básicos atendidos por personal auxiliar ${ }^{(15)}$.

En setiembre de 1983, el Ministerio de Salud del Perú aprobó en la Norma de Control de Crecimiento y Desarrollo Infantil basada en el uso del Carné de Crecimiento y Desarrollo y la Ficha de Crecimiento y Desarrollo para niños y niñas, mediante gráficas peso/ edad y talla/edad en base al patrón de referencia del National Center for Health Statistics (NCHS), de EEUU. que incluía el registro de las vacunaciones y pautas para el desarrollo psicomotor. Desde entonces el control de crecimiento y desarrollo del niño se realiza en el país como una estrategia de atención integral del niño y la niña, donde se integran las otras intervenciones, de vacunación y evaluación del desarrollo psicomotor ${ }^{(16)}$. 
Desde el año 2007 estableció la implementación del enfoque de Presupuesto por Resultados, que tiene como principal instrumento operativo a los Programas Presupuestales considerados en la ley del Presupuesto Fiscal 2007. En el año 2008 se empezó a implementar. del Programa Presupuestal Articulado Nutricional (PAN), con el propósito de reducir la desnutrición crónica infantil, que tiene entre sus productos el "Control de Crecimiento y Desarrollo completo para la edad" y "Municipios que promueven el cuidado infantil(15).

$\mathrm{Y}$, por último, en este contexto, la oferta de nuestros servicios de salud implementados en las Instituciones Prestadoras de Salud tiene más de 35 años en el marco de la Atención Primaria de Salud, que tiene como propósito fundamental la vigilancia y el crecimiento y desarrollo de grupos vulnerables especialmente los niños menores de cinco años de edad. Por lo que el Ministerio de Salud (MINSA), ha ido implementando una serie de estrategias enmarcadas en la Atención Integral del Niño (a), que forman parte de las políticas de salud bajo el enfoque de Presupuesto por Resultado (PpR), donde se busca garantizar una atención de calidad en Instituciones Prestadoras de Salud, teniendo como prioridad el niño(a), donde los resultados son monitoreados de manera permanente, orientados a la disminución de enfermedades prevalentes como la desnutrición crónica, anemia, enfermedades inmunoprevenibles y otros asociados con las condiciones de vida del niño(a); por lo que a nivel operativo se debe garantizar la permanencia de un profesional de enfermería competente y la dotación de recursos e insumos para ejecutar las actividades programadas. Lo que finalmente redunda en el cumplimiento de los Indicadores Trazadores ${ }^{(17)}$.

Por tal motivo en el presente estudio se busca analizar la relación entre la concentración y la periodicidad del control y el perfil de crecimiento y desarrollo en niños menores de un año del Centro de Salud Aparicio Pomares de Huánuco, durante el 2018.

\section{MÉTODOS}

\section{Tipo de estudio}

Observacional, longitudinal, retrospectivo y analítico. El enfoque fue cuantitativo, y el nivel relacional. El diseño del estudio fue correlacional.

\section{Población}

Estuvo respaldada por 309 historias clínicas de niños menores de un año que han cumplido 11 meses de edad en el año 2017.

\section{Instrumentos de medición}

Se elaboró una ficha de recolección de datos para la revisión de las historias clínicas integrales del niño sobre la evaluación del perfil de crecimiento y desarrollo y el control de crecimiento y desarrollo del recién nacido y del niño de 1 a 11 meses de edad.

Respecto a la validación del instrumento, en primer lugar se realizó la validación cualitativa, mediante la validez de contenido del juicio de cinco expertos, quienes de forma unánime apreciaron en forma satisfactoria y sin observaciones. Luego, se realizó una prueba piloto en una muestra de 10 niños con 11 meses de edad, atendidos en el Centro de Salud Aparicio Pomares durante periodo del 2018, donde se pudo poner a prueba su aplicación, definiendo en forma definitiva la confección del instrumento.

\section{Procedimiento}

Se realizaron las coordinaciones pertinentes para la autorización del estudio, el cual consto de una solicitud de permiso, siendo recepcionada y aceptada el 16 de agosto del presente año. Asimismo, los recursos materiales y de servicio fueron abastecidos y financiados por la Universidad de Huánuco. Luego, se realizó una capacitación sobre los objetivos del estudio tanto para la prueba piloto como para la recolección propiamente dicho, para ello se contrataron los servicios de dos trabajadores administrativos (técnicos en estadística) con una experiencia mayor a 5 años en el campo de la salud, para la realización de la recolección de datos. Por último, la aplicación de los instrumentos se efectuó mediante la ficha de recolección de datos, siendo la fuente directa las historias clínicas de los niños en estudio comprendidos entre en 1 al 31 de diciembre de 2017, la cual se desarrolló del 15 al 31 de octubre del 2018, en los turnos de mañana y tarde; siendo el promedio de recojo de información por cada ficha de 15 minutos.

\section{Análisis de datos}

En el análisis descriptivo de los datos se utilizaron estadísticas de frecuencia y de porcentaje, como las medidas de tendencia central y de dispersión.

En la comprobación de la hipótesis se utilizó la Prueba de chi-cuadrada de Pearson de independencia, con una probabilidad de significancia de 0,05\%. En todo el procesamiento de los datos se utilizó el paquete estadístico SPSS en su versión 22,0.

\section{RESULTADOS}

Dentro de las características generales, encontramos que un $54,4 \%$ (168 niños) fueron del sexo masculino y $45,6 \%$ (141 niños) de sexo femenino. 
Respecto a los controles, se observa que según transcurren los controles estos disminuyen, donde en el recién nacido $79,2 \%$ (221 niños) acaban con cuatro controles y asimismo, en el niño menor de un año el 69,3 \% (214 niños) terminan con 11 controles (ver figura 1).

En relación al promedio de días de asistencia según controles, evidenciamos que en niños recién nacidos el promedio de días de asistencia fueron diferente a los días de control; los cuales serían 2, 7, 14 y 21 días. De igual manera, en el niño menor de un año los días promedio de asistencia son distantes de un control cada 30 días (ver tabla 1).

Concerniente a la concentración de controles, en niños recién nacidos, se encontró que 74,2 \% (207 niños) presentaron concentración y en niños menores de un año 62,8 \% (194 niños) tuvieron concentración (ver figura 2).

En cambio, relativo a la periodicidad de controles, en niños recién nacidos, se halló que 43,0 \% (120 niños) tuvieron la periodicidad y en niños menores de un año $13,9 \%$ (43) mantuvieron la periodicidad (ver figura 3).

Con respecto al crecimiento, $94,3 \%$ tuvieron crecimiento adecuado en recién nacidos y $57,0 \%$ presentaron crecimiento adecuado en menores de un año. Y, en cuanto al desarrollo psicomotor, 97,7 \% de niños menores de un año presentaron desarrollo psicomotor normal.

En cuanto al análisis inferencial de los resultados, encontramos que la concentración de controles se relaciona significativamente con el crecimiento de recién nacidos, con $p \leq 0,004$ y en niños menores de un año, la concentración de controles se relaciona

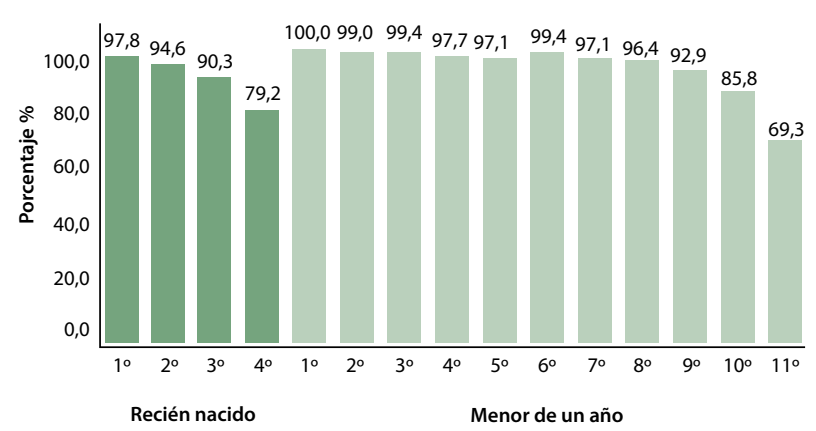

Figura 1. Controles de niños menores de un año del Centro de Salud Aparicio Pomares,Huánuco, 2017
Tabla 1. Promedio de días de asistencia según controles de niños recién nacidos del Centro de Salud Aparicio Pomares, Huánuco, 2017

\begin{tabular}{|c|c|c|c|c|}
\hline \multirow{2}{*}{ Control } & \multicolumn{4}{|c|}{$n=309$} \\
\hline & Frecuencia & Media & Mínimo & Máximo \\
\hline \multicolumn{5}{|c|}{ Recién nacido } \\
\hline Primero & 273 & 5,5 & 1 & 24 \\
\hline Segundo & 264 & 9,8 & 2 & 23 \\
\hline Tercero & 252 & 16,5 & 11 & 29 \\
\hline Cuarto & 221 & 22,0 & 14 & 30 \\
\hline \multicolumn{5}{|c|}{ Menor de un año } \\
\hline Primero & 309 & 34,1 & 25 & 127 \\
\hline Segundo & 306 & 31,1 & 7 & 117 \\
\hline Tercero & 304 & 31,5 & 4 & 134 \\
\hline Cuarto & 300 & 33,3 & 4 & 245 \\
\hline Quinto & 293 & 33,0 & 14 & 120 \\
\hline Sexto & 299 & 33,0 & 15 & 100 \\
\hline Septimo & 300 & 33,2 & 5 & 99 \\
\hline Octavo & 294 & 33,5 & 7 & 112 \\
\hline Noveno & 285 & 32,3 & 4 & 88 \\
\hline Décimo & 264 & 33,3 & 2 & 91 \\
\hline Undécimo & 212 & 31,6 & 3 & 67 \\
\hline
\end{tabular}

significativamente con el crecimiento $(p \leq 0,003)$ y desarrollo psicomotor $(p \leq 0,014)$ (ver tabla 2,3 ).

\section{DISCUSIÓN}

Al respecto, numerosos estudios muestran concordancia con nuestros hallazgos y confirman lo señalado.

Así tenemos un análisis con información básica presentado en "The Lancet: Maternal and Child Nutrition Series" (2013) y citado por la Secretaría Técnica del Plan Toda una Vida, que permite entender que al alargar los controles de salud en niños y niñas por encima del $90 \%$, se lograría una disminución de la desnutrición crónica infantil hasta en $20 \%$ y también en $15 \%$ el índice de mortalidad infantil ${ }^{(7)}$.

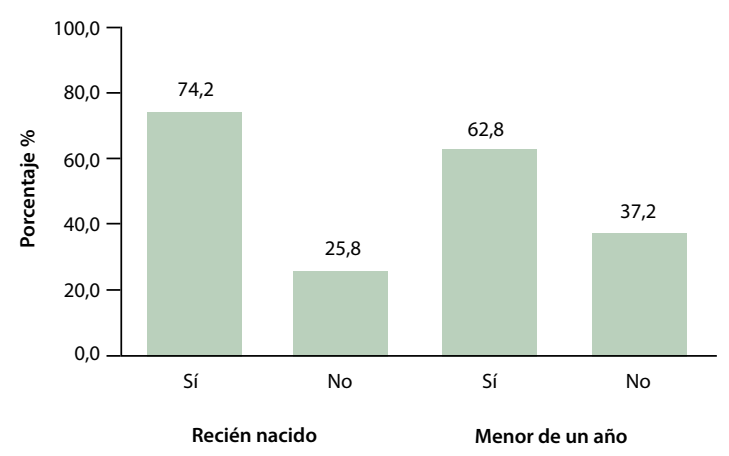

Figura 2. Concentración de controles de niños menores de un año del Centro de Salud Aparicio Pomares,Huánuco, 2017 


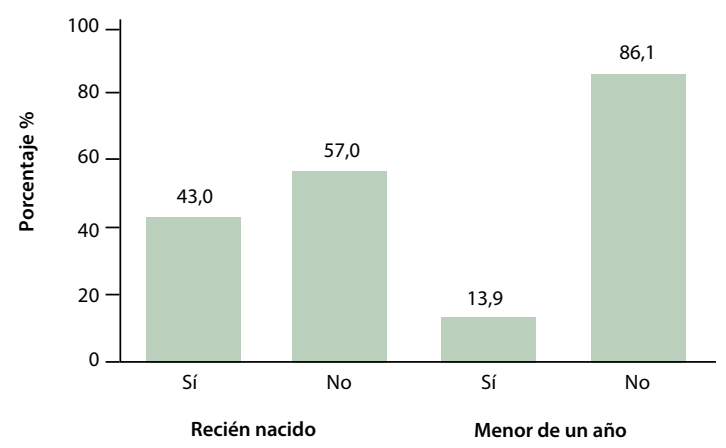

Figura 3. Periodicidad de controles de niños menores de un año del Centro de Salud Aparicio Pomares, Huánuco, 2017

Asimismo, Mamani ${ }^{(18)}$ logró establecer una significancia estadística entre la asistencia al control de crecimiento y desarrollo con el estado nutricional en la etapa de lactancia; además, mostrando que hay relación entre la asistencia al control de crecimiento y desarrollo y el estado nutricional a través de indicadores antropométricos peso /edad en lactantes del Puesto de Salud Progreso I-2, San Juan Bautista, en Iquitos.

Jaillita ${ }^{(19)}$ concluyó que la asociación entre los controles de crecimiento y desarrollo y la desnutrición crónica infantil fue negativa y la asociación entre vacunas completas según edad y la desnutrición crónica también fue negativa. También informó que los controles de crecimiento y desarrollo fueron más significativos que el producto vacunas completas.

Igualmente, Martínez y Palma ${ }^{(20)}$ pusieron en evidencia un listado de intervenciones costo-efectivas clasificadas en dos tipos: específicas y sensibles, para luchar contra el fenómeno de la desnutrición crónica y el déficit de micronutrientes. Dentro de las intervenciones específicas se encuentran: lactancia materna y alimentación complementaria para niños de 6 a 23 meses de edad, suplementación con micronutrientes, fortalecimiento de alimentos de consumo masivo para prevenir el déficit de micronutrientes y estrategias de educación y

Tabla 3. Relación entre concentración y periodicidad de controles y el desarrollo psicomotor de niños menores de un año del Centro de Salud Aparicio Pomares, Huánuco, 2017

\begin{tabular}{lcccc}
\hline \multirow{3}{*}{ Controles } & \multicolumn{2}{c}{ Desarrollo psicomotor } & & \\
\cline { 2 - 3 } & $\begin{array}{c}\text { Normal } \\
\%\end{array}$ & $\begin{array}{c}\text { Trastorno del } \\
\text { desarrollo } \\
\%\end{array}$ & $\begin{array}{c}\text { Chi } \\
\text { cuadrada }\end{array}$ & Valor - p \\
\hline Concentración & 61,8 & 1,0 & 8,55 & 0,014 \\
Periodicidad & 13,6 & 0,3 & 1,59 & 0,456 \\
\hline
\end{tabular}

Tabla 2. Relación entre concentración y periodicidad de controles y el crecimiento de niños menores de un año del Centro de Salud Aparicio Pomares, Huánuco, 2017

\begin{tabular}{|c|c|c|c|c|}
\hline \multirow[b]{2}{*}{ Controles } & \multicolumn{2}{|c|}{ Crecimeinto } & \multirow{2}{*}{$\begin{array}{l}\text { Prueba } \\
\text { Chi } \\
\text { cuadrada }\end{array}$} & \multirow[b]{2}{*}{ Valor - p } \\
\hline & $\begin{array}{c}\text { Adecuado } \\
\%\end{array}$ & $\begin{array}{c}\text { Inadecuado } \\
\%\end{array}$ & & \\
\hline \multicolumn{5}{|l|}{ Recién nacido } \\
\hline Concentración & 71,7 & 2,5 & 8,22 & 0,004 \\
\hline Periodicidad & 40,5 & 2,5 & 0,00 & 0,951 \\
\hline \multicolumn{5}{|l|}{$\begin{array}{l}\text { Menor de un } \\
\text { año }\end{array}$} \\
\hline Concentración & 39,8 & 23,0 & 8,83 & 0,003 \\
\hline Periodicidad & 7,8 & 6,1 & 6,1 & 0,870 \\
\hline
\end{tabular}

promoción de la salud. En las intervenciones sensibles sobresalen las vacunas, la desparasitación, la promoción del lavado de manos, el control neonatal y los programas de estimulación temprana.

Tafur (21) concluyó que existe un impacto positivo en la reducción de la desnutrición crónica infantil a partir de la implementación del programa articulado nutricional, debido a que existió un aumento significativo del control del crecimiento y el desarrollo y la suplementación de hierro en niños menores de 3 años.

Enriquez (22) llegó a la conclusión que las intervenciones efectivas cimentadas en la prevención y promoción para la reducción de la desnutrición crónica fueron: el porcentaje de niños menores de 36 meses con controles de crecimiento y desarrollo completo, el porcentaje de niños menores de 36 meses con vacunas básicas completas y el porcentajes de viviendas con acceso al agua tratada.

Cruzado ${ }^{(23)}$ logró identificar que la intervención del Programa Articulado Nutricional disminuye la posibilidad de ser desnutrido y no obstante incrementa la posibilidad de poseer sus controles de crecimiento y vacunas completas. Por tanto, existe una asociación negativa y significativa entre el Programa Articulado Nutricional y la desnutrición crónica en niños.

También, algunas experiencias latinoamericanas relevantes en el descenso de la desnutrición crónica infantil como Chile y Cuba, que iniciaron con niveles de desnutrición crónica infantil semejantes al resto del continente, consiguieron en dos décadas reducciones sustanciales, a través de un grupo de intervenciones implementadas en la atención primaria de salud, en las que el control periódico del crecimiento de los niños fue el eje integrador ${ }^{(24)}$. 
La investigación realizada no está exenta de limitaciones. En primer lugar, estas limitaciones se deben al diseño transversal del estudio; el cual no permitió presentar explicaciones causales, sino asociaciones. Sin embargo permite generar preguntas hacia la causalidad para futuros estudios, como por ejemplo: ¿Cuál es la influencia del control de crecimiento $y$ desarrollo en el crecimiento $y$ desarrollo de niños menores de 3 años, ¿Cuál es la implicancia del control de crecimiento y desarrollo en la oportunidad de administración de las vacunas para la edad?, ¿Cuál es la tasa de deserción del control de crecimiento y desarrollo asociado a la prevalencia de enfermedades en la infancia (anemia, parasitosis, infecciones respiratorias agudas y enfermedades gastro intestinales).

Por último, como futuras líneas de investigación, en primer lugar sería conveniente tratar de cumplir la limitación expuesta anteriormente. Se concluye que la concentración de controles se relaciona significativamente con el crecimiento de recién nacidos en estudio, con un $\mathrm{p} \leq 0,004$. Asimismo, la concentración de controles se relaciona significativamente con el crecimiento de niños menores de un año (de 01 a 11 meses), con $p \leq 0,003$. La concentración de controles se relaciona significativamente con el desarrollo de niños menores de un año (01 a 11 meses), con $p \leq$ 0,014 . Sin embargo, la periodicidad de controles no se relaciona con el crecimiento de recién nacidos en estudio, con $\mathrm{p}>0,05$. Por su parte la periodicidad de controles no se relaciona con el crecimiento de niños menores en estudio, con $p>0,05$. Mientras que la periodicidad de controles no se relaciona con el desarrollo de niños menores en estudio, con $p>0,05$.

\section{REFERENCIAS BIBLIOGRÁFICAS}

1. Spears D. ¿Cuánta variación internacional en la altura de los niños puede explicar el saneamiento? Washington D. C.: Banco mundial; 2013.

2. Fondo de las Naciones Unidas para la Infancia (UNICEF). Mejora de la nutrición infantil: el imperativo alcanzable para el progreso global. New York; 2013.

3. UNICEF, OMS, Banco Mundial. Estimaciones conjuntas de desnutrición infantil: niveles y tendencias. Ginebra; 2016 [internet]. [Consultado 22 noviembre 2018] Disponible en: http://data.unicef.org/nutrition/malnutrition.html

4. Roldan JA, Carrasco R, Guarneros N. El hambre, parte de la cultura en los hogares marginales de México. Los casos de la Huasteca Potosina y la Sierra Tarahumara. Editorial Académica Española; 2012.

5. Gutiérrez JP, Rivera-Dommarco J, Shamah-Levy T, Villalpando-Hernández S, Franco A, Cuevas-Nasu L, et al. Encuesta Nacional de Salud y Nutrición 2012. Resultados
Nacionales. Cuernavaca, México: Instituto Nacional de Salud Pública; 2012.

6. Programa de Naciones Unidas para el Desarrollo (PNUD). Objetivos de Desarrollo del Milenio. Colombia: PNUD; 2014. [internet]. [Consultado octubre 2018]. Disponible en: http://www.undp.org/content/dam/undp/library/ MDG/english/MDG\%20Country\%20Reports/Colombia/informeanualodm2014.pdf.

7. ENSANUT. Encuesta Nacional de Salud y Nutrición - ENSANUT [internet]. 2012. [Consultado noviembre 2018]. Disponible en: http://www.salud.gob.ec/encuesta-nacional-de-salud-y-nutricion-ensanut/.

8. Instituto Nacional de Estadística e Informática (INEI). Resultados de la Encuesta Demográfica y de Salud Familiar, ENDES 2017. Lima: INEI; 2018.

9. Direccion Regional de Salud, DIRESA. Sistema de Información del Estado Nutricional, SIEN. Huánuco: DIRESA; 2018.

10. Fondo de las Naciones Unidas para la Infancia. Estado mundial de la infancia 1998. Ginebra: UNICEF; 1998.

11. Smith L, Haddad L. Superar la desnutrición infantil en los países en desarrollo: logros pasados y elecciones futuras. Washington: International Food Policy Research Institute; 2000.

12. Victoria C, Adair L, Fall C, Hallal P, Martorell R, Richter L, Sachdev HS. Desnutrición materna e infantil: consecuencias para la salud de los adultos y el capital humano. Lancet. 2008;371(9609):340-57.

13. Martins VJB, Toledo Florêncio TMM, Grillo LP, et al. Efectos duraderos de la desnutrición. Int J Env Res Pub He. 2011 Jun;8(6):1817-1846.

14. Moreno-Ruiz DV, Picón MM, Marrugo-Arnedo CA, Verena Marrugo A, Alvis Guzmán NR. Determinantes socioeconómicos del estado nutricional en menores de cinco años atendidos en el Hospital Infantil Napoleón Franco Pareja. Rev Univ Ind Santander Salud. 2017;49(2):352-363.

15. Castillo O, Zúñiga R. Evaluación de diseño y ejecución presupuestal de la intervención pública "CRED y Municipios Saludables". Lima: Ministerio de Salud; 2015.

16. Resolución Ministerial $N^{\circ} 0136-84-S A / D V M$.

17. Huamán $E E$, Huingo $C D$. Control de crecimiento y desarrollo del menor de un año por el profesional de enfermería en el Centro de Salud Pachacutec-Cajamarca, 2015. [Tesis de licenciatura]. Cajamarca: Universidad Privada Antonio Guillermo Urrelo; 2016.

18. Mamani DK. Asistencia al control de crecimiento, desarrollo y el estado nutricional en lactantes del puesto de salud progreso i-2, san juan bautista, 2016. [Tesis de licenciatura]. Iquitos: Universidad Privada de la Selva Peruana; 2017.

19. Jaillita SS. Análisis del Programa Articulado Nutricional en el año 2012 a través de los productos controles de crecimiento y desarrollo (CRED) completo y vacunas completas. [Tesis de licenciatura]. Lima: Universidad Peruana De Ciencias Aplicadas; 2014.

20. Martínez R, Palma A. Cerrando la brecha. Modelo para estimar el costo de erradicar la desnutrición crónica y las deficiencias de micronutrientes. CEPAL - Serie Manuales $\mathrm{N}^{\circ}$ 84. Chile, 2015.

21. Tafur YJ. Impacto del programa articulado nutricional en la desnutrición crónica infantil a nivel nacional, según la encuesta demográfica de salud familiar, 2008-2016. 
[Tesis de especialidad]. Lima-Perú: Universidad de San Martín de Porres; 2018.

22. Enriquez JA. Impacto del programa presupuestal articulado nutricional en la desnutrición crónica infantil en menores de 5 años, en la Unidad Ejecutora GERESA Lambayeque en el periodo 2012-2016. [Tesis de Maestría]. Chiclayo-Perú: Universidad Cesar Vallejo; 2017
23. CruzadoV. Análisis del impacto del Programa Presupuestal Articulado Nutricional (PAN) sobre la desnutrición crónica en niños menores de 5 años. Documento de Trabajo. Dirección General de Presupuesto Público. Ministerio de Economía y Finanzas; 2012.

24. Conferencia Ministerial Regional, Capítulo II. La erradicación de la desnutrición infantil en Chile. WFP, 2008. 